\title{
Design and Simulation of a Low Power Amplifier for CMOS MEMS Sensors Using Wide Swing Cascode Current Mirror
}

\author{
Muhammad Umer Mian ${ }^{1}$, John Ojur Dennis ${ }^{2}$, M Haris Bin Mohd Khir ${ }^{1} \&$ Nebyu Yonas Sutri ${ }^{1}$ \\ ${ }^{1}$ Department of Electrical and Electronic Engineering, Universiti Teknologi PETRONAS, Bandar Sri Iskandar, \\ Perak, Malaysia \\ ${ }^{2}$ Department of Fundamental and Applied Sciences, Universiti Teknologi PETRONAS, Bandar Sri Iskandar, \\ Perak, Malaysia \\ Correspondence: Muhammad Umer Mian, Department of Electrical and Electronic Engineering, Universiti \\ Teknologi PETRONAS, Bandar Sri Iskandar, Perak, Malaysia. Tel: 60-193-779-462. E-mail: \\ umermian@gmail.com
}

Received: October 8, 2013

Accepted: November 11, $2013 \quad$ Online Published: November 22, 2013

doi:10.5539/mas.v7n12p71

URL: http://dx.doi.org/10.5539/mas.v7n12p71

\begin{abstract}
We present a low power amplifier integrated with a wide swing cascode current mirror (WSCCM) based on PMOS transistors. To derive the low power amplifier a current of $120 \mathrm{uA}$ is required, which is achieved by varying the widths of the transistors. The best result is achieved by varying the widths up to 6um with an output saturated current response of $120.04 \mathrm{uA}$. SECTPRE of CADENCE design tool is used as the design software to carry out the simulations. The proposed circuits are designed and verified in MIMOS 0.35 um CMOS and BSIM3 transistor model technology. The low power amplifier achieves an output response of $44.71 \mathrm{mV}$ with a minimum power consumption of $0.485 \mathrm{~mW}$.
\end{abstract}

Keywords: CMOS, current mirror, amplifier, cascode configuration

\section{Introduction}

MEMS integration with CMOS is the next evolutionary step in micro-technology (Gusev et al., 2010). The integration of both processes CMOS and MEMS is achieved by two techniques, hybrid and monolithic. The hybrid technique involves bonding of two wafers using bonding wires. The hybrid technique allows independent optimization of both the MEMS and CMOS components but the technique is not a cost effective solution in terms of packaging and assembly. The monolithic CMOS MEMS technique on the other hand increases the reliability of the overall system as it eliminate the number of off chip connections and bonding wires thus reducing parasitic effects.

The major disadvantages of monolithic CMOS MEMS integration include fabrication limitation and noise. Many MEMS sensors these days are based on capacitive sensing technique, scaling down is another challenge for these MEMS sensors when monolithically integrated on the same die. Sensors cannot be smaller than its sensitive components. Due to MEMS requirement of surface micromachining, all sensing capacitive components of sensors are located at the surface. The physical limitation of the sensors reduces the capacitance values in range of femto Farads, which bring up another challenge for the designers to design a highly sensitive capacitance detection circuitry as readout for these sensors (John \& David, 2003).

In this paper we present a low power amplifier integrated with a PMOS based wide swing cascode current mirror as a sense detection circuitry for CMOS MEMS monolithic sensors. The capacitance range of CMOS MEMS sensors are generally in femto farad giving output sense signal up to few milli Volts. With a $6 \mathrm{mVpp}$ test signal, the low power amplifier achieves a stable output with minimum power consumption through width adjustments of the MOS devices.

\section{WSCCM Architecture}

A current mirror is one of the basic and important building blocks of analog integrated circuits (Tseng, Meng, \& Huang, 2007). The purpose of current mirrors is to perform current amplification, active loading, signal level shifting and biasing (Azhari, Hassan, \& Khalil, 2011). An ideal current mirror can be defined as an amplifier which ideally inverts the current or control it as a current-controlled current source (CCCS) (Hitesh \& Goel, 
2012). Various architecture schemes have been reported for current mirrors (Sampietro, Bertuccio, \& Fasoli, 2000; Sarao, Wang, Wu, \& Kwok, 2002; Tseng et al., 2007; Kim, Yun, K. Lee, \& S. Lee, 2008; Azhari et al., 2011; Petrellis, George, \& Dimitrios, 2012).

The architecture of current mirror is employed in cascode configuration to achieve characteristics such as current matching and high impedance at output and input stages. The useful aspect of the architecture is that it increases the resistance of the overall circuit by a factor of $g_{m} r_{o}$ where $g_{m}$ is the transconductance and $r_{o}$ is the output resistance of the MOSFET (Bhawna, Maneesha, \& Gupta, 2013; Allen \& Holberg, 2004). These high performance characteristics of cascode current mirror achieves high priority over simple current mirrors as a choice for complex circuits such as variable gain low noise amplifiers etc (Yang, Kim, Yun, Y. Lee, \& J. Lee, 2008; Eldbib \& Musil, 2008; Laajimi \& Masmoudi, 2012). Higher input output compliance voltages of the simple cascode current mirror provides a drawback for its selection in complex circuits (Toth \& Meijer, 1992). The topology given in Figure 1(a) is an alternate topology for high swing cascode as it provides better performance by maintaining the same output resistance when compared to a standard cascode topology. Figure 1(a) topology also provides lower compliance voltage when compared with standard cascode structure.

The architecture of a high swing current mirror is based on a biasing circuit and a cascode current source (Shukla, Devashrayee, \& Naik, 2011). Biasing is achieved by the two terminals in the current source, the rest of circuit employs 4 transistors Q1, Q2, Q3 and Q4. The fifth transistor Q5 ensures all the other transistors remain in saturation region for better performance of the circuit (Aziz, Mamun, Bhuiyan, \& Bakar, 2013). WSCCM output impedance is kept high for high output swing. The small signal analysis behaviour of WSCCM is quite similar to the basic cascode, whereas biasing technique is achieved quite differently. The total drain to source voltage of Q2 and Q4 defines the biasing voltage which is usually kept at minimum value (Nojdelov \& Nihtianov, 2009). Bias voltage and drain to source voltage of Q2 and Q4 contributes to the total output voltage. The widths and length ratio of the MOSFETs, always keep the transistor Q3 in on state and at the edge of saturation. The transistor Q5 added in series ensures the drain voltages of the other transistors to be identical and also remove any error caused by channel length modulation factor (Rosli, Mamun, Bhuiyan, \& Husain, 2012).

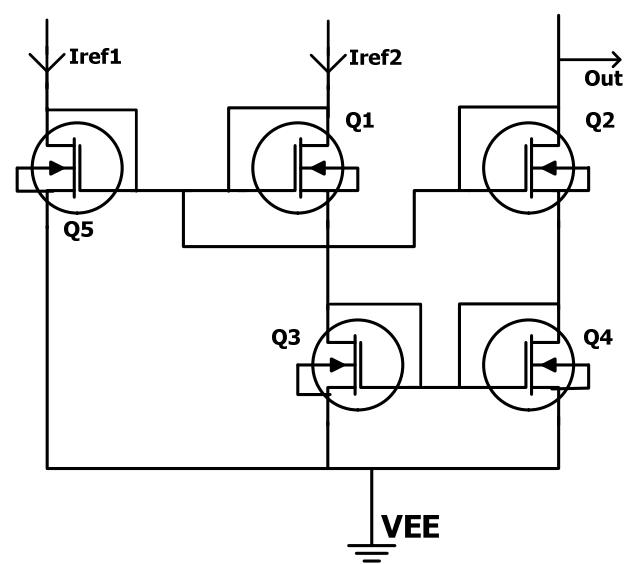

(a)

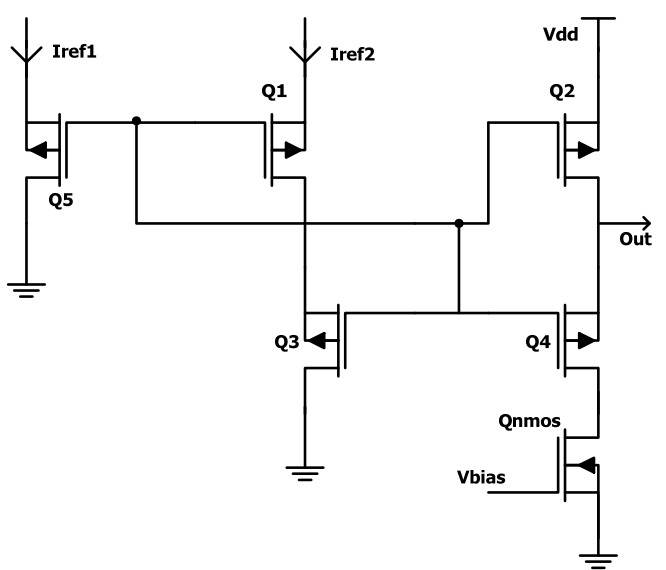

(b)

Figure 1. Typical wide swing cascode current mirror vs proposed PMOS based WSCCM

\section{Proposed PMOS Based WSCCM}

The proposed WSCCM is PMOS based with one NMOS transistor added to the circuit as a source follower. The WSCCM is employed as a current source in replacement to current sink using NMOS transistors which were being used conventionally. The proposed PMOS based WSCCM is given in Figure 1 (b).

The simulation of the proposed WSCCM is carried out using 0.35um CMOS and BSIM3 transistors models in CADENCE design tool software. WSCCM outputs with varying transistor widths are shown in Figure 2 (a).

The simulations show that with increasing transistor widths, WSCCM achieves better performance. Figure 2 (b) shows the most stable current output achieving $120.04 \mathrm{uA}$ while achieving a quicker response time in the saturation region when compared to other outputs. The results for 6 different transistor widths are summarized in Figure 3. The transistor widths for 6 transistors used in the proposed WSCCM are given in Table 1. 


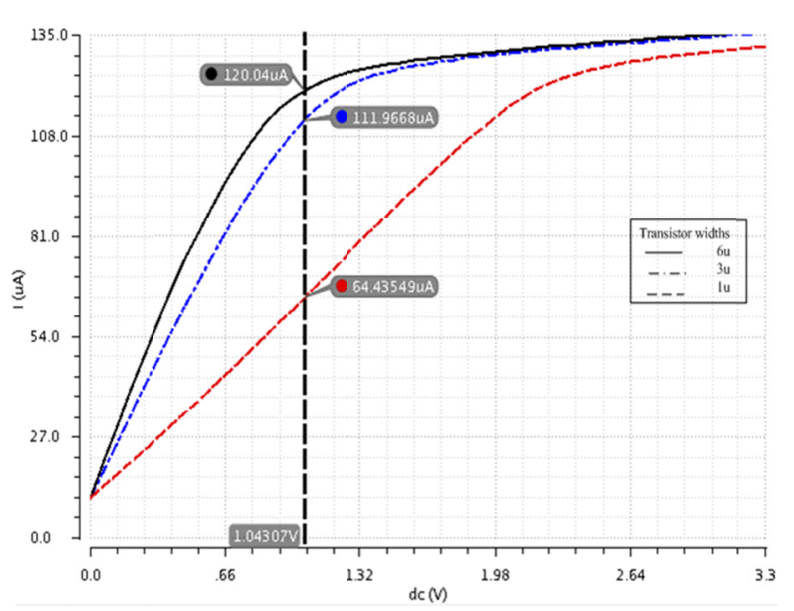

(a)

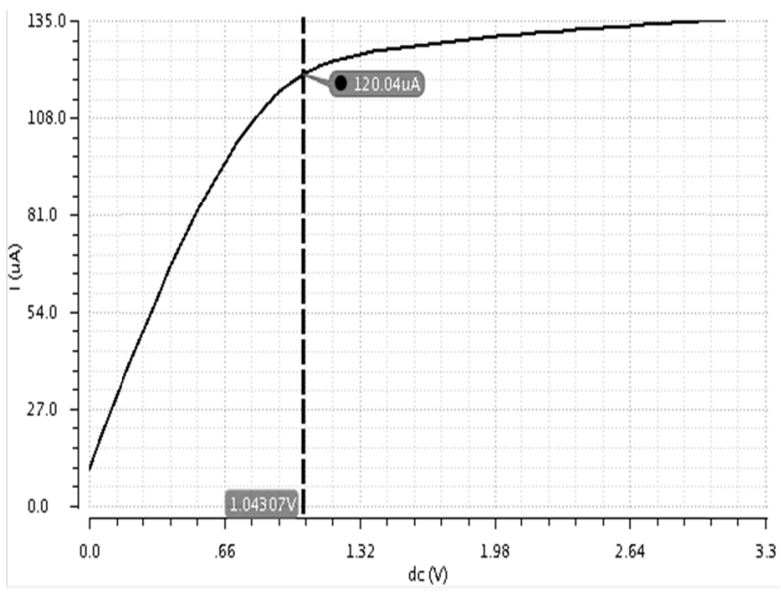

(b)

Figure 2. (a) Current output of WSCCM with multiple transistor widths, (b) desired output at 6um width

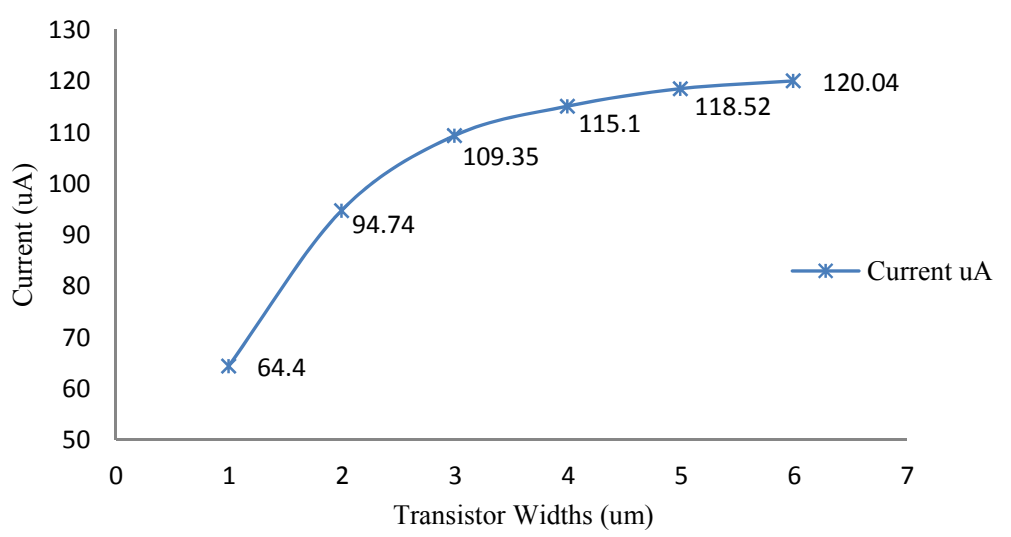

Figure 3. WSCCM transistor widths and their corresponding output currents

Table 1. Lengths and widths of proposed WSCCM

\begin{tabular}{cll}
\hline Transistors & Widths & Lengths \\
\hline T1, T2, T3, T4 & $6.0 \mathrm{um}$ & $0.35 \mathrm{um}$ \\
T5 & $0.9 \mathrm{um}$ & $0.35 \mathrm{um}$ \\
T6 (NMOS) & $0.65 \mathrm{um}$ & $0.35 \mathrm{um}$ \\
\hline
\end{tabular}

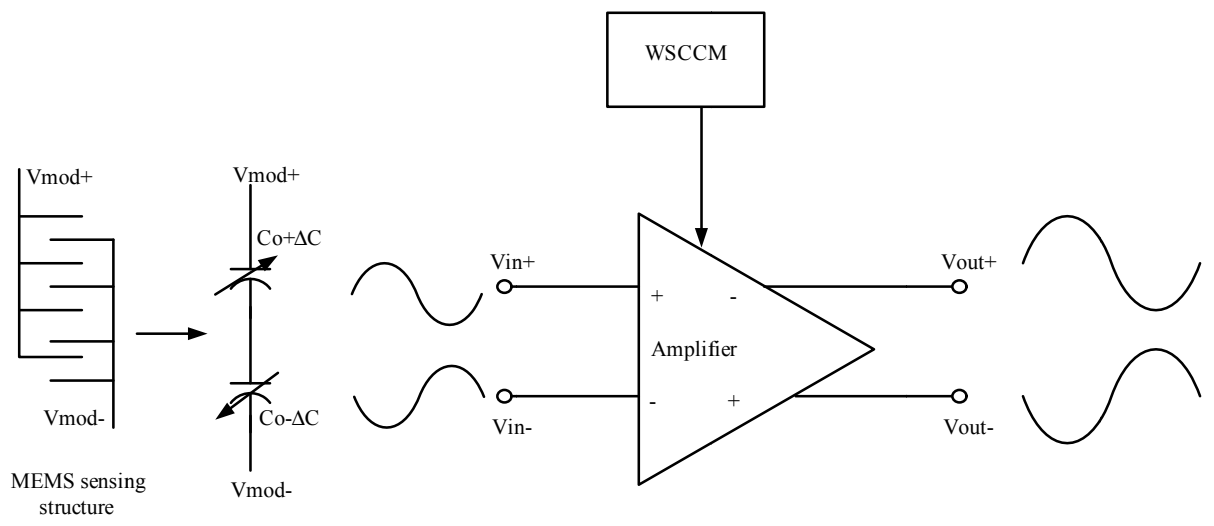

Figure 4. Block diagram of the overall system 


\section{WSCCM Integration with Low Power Amplifier}

Low power cascode amplifiers are used for differential capacitive sensing in many MEMS sensors and require stable biasing current sources. A block diagram representing the overall system is shown in Figure 4. The proposed PMOS based WSCCM is used as a stable and continuous current source with low power amplifier as shown in Figure 5. A stable 126.16 uA current input is achieved for the low power amplifier resulting in a 44.71 $\mathrm{mV}$ output with $6 \mathrm{mVpp}$ input with a gain of $17 \mathrm{~dB}$. The mechanical comb fingers designed in the MEMS sensors provide a fully differential capacitive sensing scheme as an input to the proposed amplifier. The differential scheme employs two input signals as a result of change in capacitances between the fingers. The two input sensed signals are computed by Equation 1 (Wu, 2002).

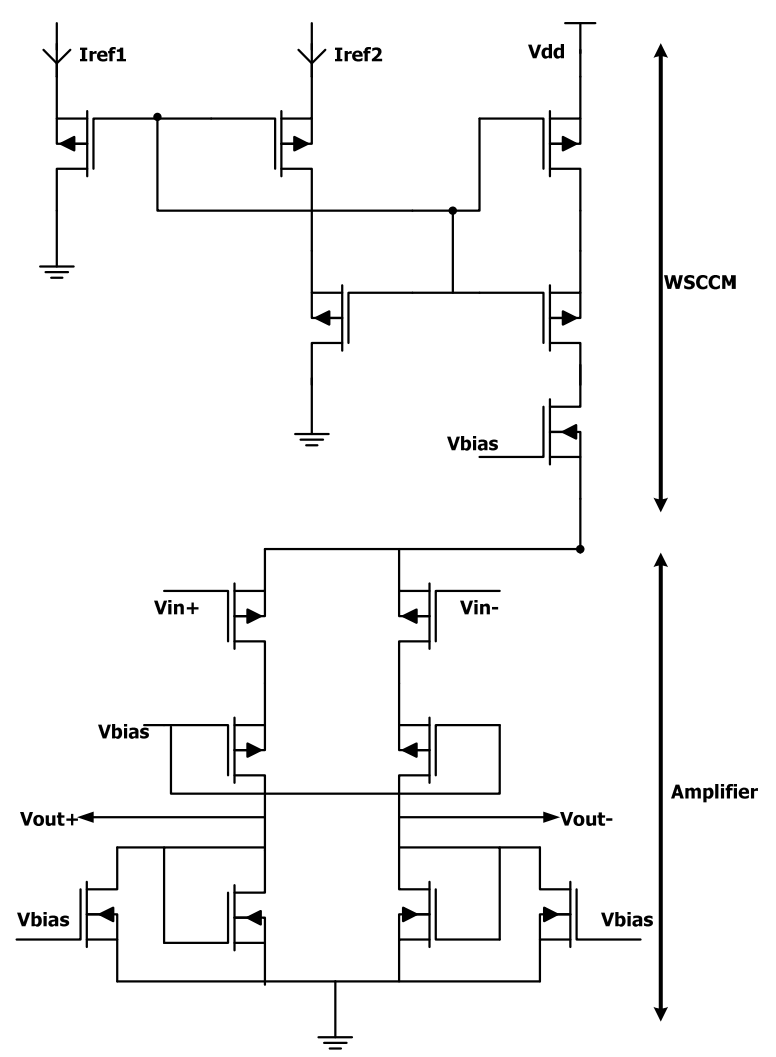

Figure 5. Integration of the proposed WSCCM with low power amplifier

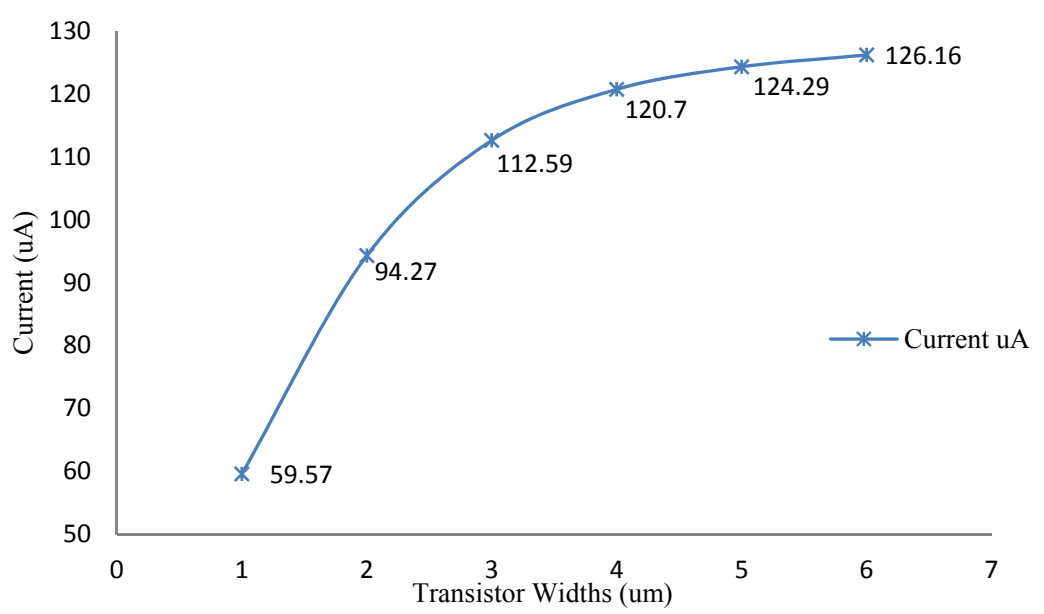

Figure 6. Current response of WSCCM-integrated amplifier vs. transistor widths of WSCCM 


$$
V_{s}=\frac{(4 \times \Delta C)}{2 C_{o}+C_{p}} V_{\bmod }
$$

where $V_{s}$ is the input sensed voltage from the differential pair, $\Delta C$ is the change in capacitance due to the movement of the comb fingers, $C_{o}$ is the initial capacitance generated between the fingers of the comb drive when there is no motion, $C_{p}$ is the parasitic capacitance and $V_{\text {mod }}$ is the modulation voltage. Output current response of WSCCM when integrated with low power amplifier with various transistor widths are given in Figure 6 and are shown in Figure 7.

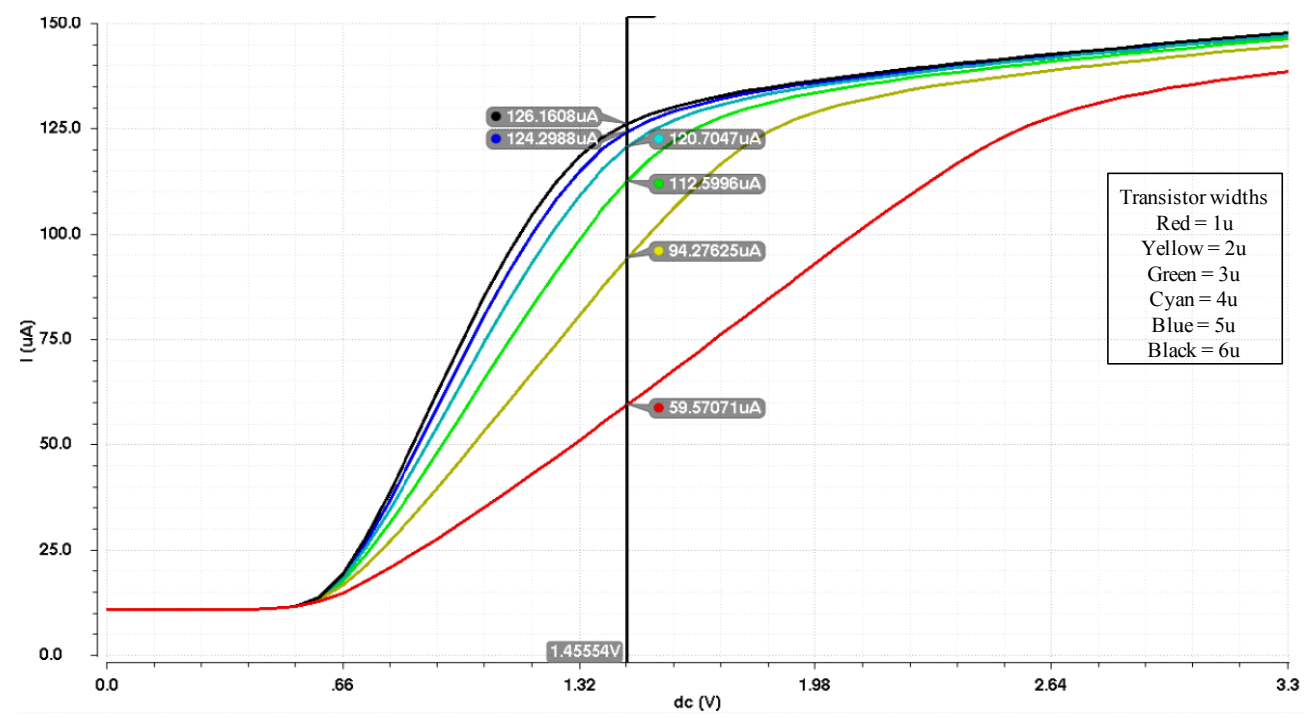

Figure 7. Current outputs for the proposed WSCCM integrated with the amplifier

Simulation result of frequency domain gain is shown in Figure 8, the amplifier when integrated with the WSCCM circuit achieves a gain of $17 \mathrm{~dB}$ at a 3-dB frequency of $23.65 \mathrm{MHz}$. With a power supply of $3.3 \mathrm{~V}$ the total power consumption is $0.485 \mathrm{~mW}$. The circuit achieves an increased bandwidth and low power consumption at a cost of slightly higher input referred noise of $92.4 \mathrm{nV} / \sqrt{ } \mathrm{Hz}$ as shown in Figure 9.

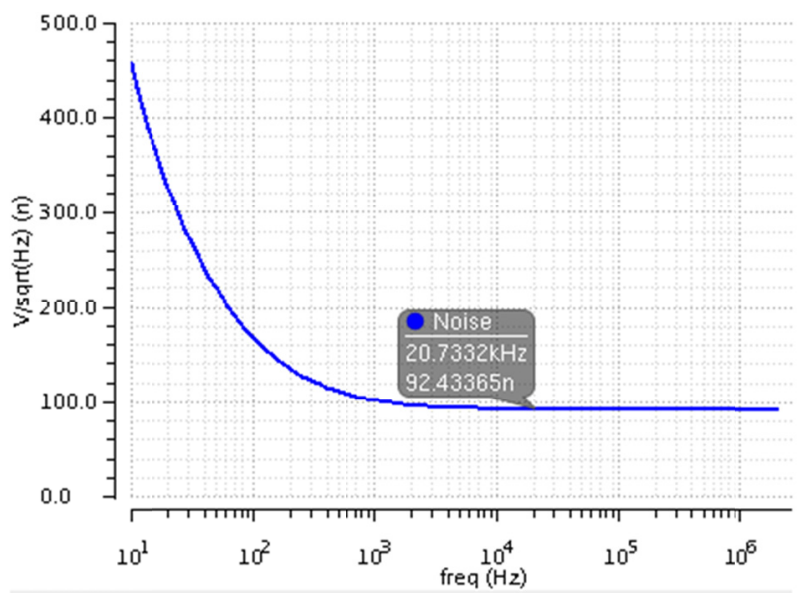

Figure 8. Gain vs frequency

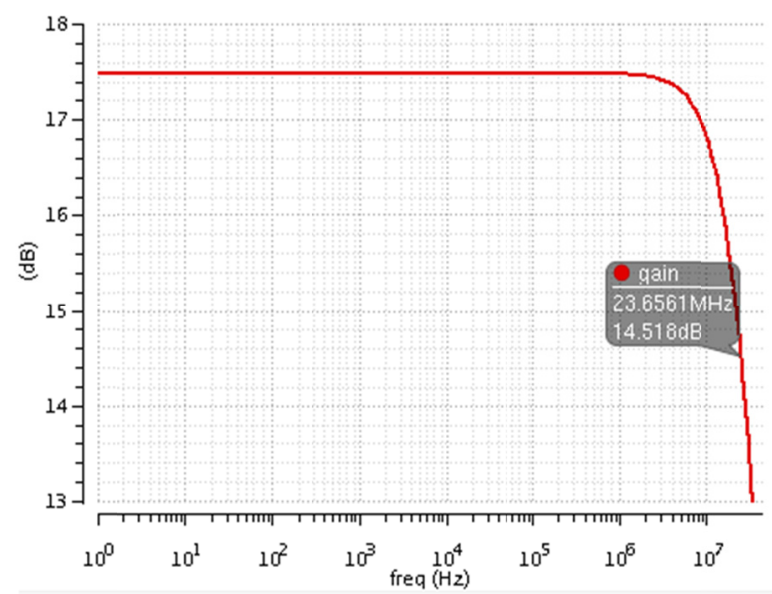

Figure 9. Input referred noise

\section{Conclusion}

The proposed PMOS based WSCCM architecture shows the best performance design ability when integrated with other circuits. The proposed design achieves a stable and continuous output current response, and provides 
a stable biasing current source for a low power amplifier which achieves a gain of $17 \mathrm{~dB}$ with a minimum power consumption of $0.485 \mathrm{~mW}$.

\section{Acknowledgment}

The authors will like to acknowledge Universiti Teknologi PETRONAS for their support and funding for this work.

\section{References}

Allen, P. E., \& Holberg, D. R. (2004). CMOS Analog Circuit Design (2nd ed.). New York: Oxford University Press.

Azhari, S. J., Hassan, F. B., \& Khalil, M. (2011). A novel ultra-high compliance, high output impedance low power very accurate high performance current mirror. Microelectronics Journal, 42(2), 432-439. http://dx.doi.org/10.1016/j.mejo.2010.10.004

Aziz, F. I. B. A., Mamun, M., Bhuiyan, M. A. S., \& Bakar, A. A. A. (2013). A Low Drop-Out Voltage Regulator in $0.18 \mu \mathrm{m}$ CMOS Technology. Modern Applied Science, 7(4), 70-76.

Bhawna, A., Maneesha, G., \& Gupta, A. K. (2013). Analysis of low voltage bulk-driven self-biased high swing cascode current mirror. Microelectronics Journal, 44(2013), 225-235. http://dx.doi.org/10.1016/j.mejo.2012.12.006

Eldbib, I., \& Musil, V. (2008). Self-cascode current controlled CCII based-tunable band pass filter. IEEE 18th International Conference on Radioelektronika (pp. 1-4).

Geen, J., \& Krakauer, D. (2003). New iMEMS ${ }^{\circledR}$ angular-rate-sensing gyroscope. Analog Dialogue, 37(3), $1-4$.

Gusev, E., Garfunkel, E., \& Dideikin, A. (2010). Advanced Materials and Technologies for Micro/Nano-Devices, 17 Sensors and Actuators. Springer. http://dx.doi.org/10.1007/978-90-481-3807-4

Hitesh, \& Goel, A. (2012). Performance Parameters of Improved Swing, Wilson \& Regulated Cascode Current Mirrors. International Journal of Advanced Research in Computer Science and Software Engineering, 2(3), 308-310.

Kim, J. Y., Yun, J. K., Lee, Y. K., \& Lee, J. S. (2008). A highly accurate BiCMOS cascode current mirror for wide output voltage range. In the Proceedings of the IEEE International Symposium on Circuits and Systems (ISCAS 2008) (pp. 2314-2317).

Laajimi, R., \& Masmoudi, M. (2012). High-performance CMOS current mirrors: applica- tion to linear voltage-to-current converter used for two-stage operational amplifier, Circuits Syst. Int. J. Sci. Res., 3, 311-316. http://dx.doi.org/10.4236/cs.2012.34044

Nojdelov, R., \& Nihtianov, S. (2009). Capacitive-sensor interface with high accuracy and stability. IEEE Transactions on Instrumentation and Measurement, 58(5), 1633-1639. http://dx.doi.org/10.1109/TIM.2009.2012957

Petrellis, N., George, A., \& Dimitrios, V. (2012). A real-time self-calibrated current mirror for wide range current reference generation, signal reproduction and delaying. Microelectronics Journal, 43(3), 225-234. http://dx.doi.org/10.1016/j.mejo.2011.12.001

Rosli, K. A., Mamun, M., Bhuiyan, M. A. S., \& Husain, H. (2012). A Low Loss Wide Swing Cascode Current Mirror in 0.18- $\mu \mathrm{m}$ CMOS Technology. Journal of Applied Sciences Research, 8(8), 4096-4102.

Sampietro, M., Bertuccio, G., \& Fasoli, L. (2000). Current mirror reset for low-power BiCMOS charge amplifier. Nuclear Instruments and Methods in Physics Research Section A: Accelerators, Spectrometers, Detectors and Associated Equipment, 439(2), 373-377. http://dx.doi.org/10.1016/S0168-9002(99)00936-5

Sarao, J., Wang, Z. J., Wu, Y. L., \& Kwok, H. L. (2002). An improved regulated cascode current mirror. Solid-State Electronics, 46(2), 307-312. http://dx.doi.org/10.1016/S0038-1101(01)00290-8

Shukla, D. S., Devashrayee, N. M., \& Naik, A. (2011). Design and Development of Self Biased Cascode Circuit with its Implementation using $0.35 \mu \mathrm{m}$ Technology. International Journal on Electronics and Communication Technology, 2(2), 140-145.

Toth, F. N., \& Meijer, G. C. M. (1992). A low-cost, smart capacitive position sensor. Instrumentation and Measurement, IEEE Transactions on, 41, 1041-1044. http://dx.doi.org/10.1109/19.199446 
Tseng, S. C., Meng, C., \& Huang, G. W. (2007). High Gain CMOS Gilbert Downconverter with Wide-Swing Cascode Current-Mirror Transconductor and Load. In the Proceedings of the IEEE Antennas and Propagation Society International Symposium (pp. 4513-4516).

$\mathrm{Wu}$, J. (2002). Sensing and control electronics for Low-Mass Low-Capacitance MEMS Accelerometers. (PhD thesis) Carnegie Mellon University.

Yang, B. D., Kim, J. S., Yun, J. K., Lee, Y. K., \& Lee, J. S. (2008). A Highly Accurate BiCMOS Cascode Current Mirror for Wide Output Voltage Range. In the Proceedings of the IEEE International Symposium on Circuits and Systems (ISCAS 2008) (pp. 2314-2317). http://dx.doi.org/10.1109/ISCAS.2008.4541917

\section{Copyrights}

Copyright for this article is retained by the author(s), with first publication rights granted to the journal.

This is an open-access article distributed under the terms and conditions of the Creative Commons Attribution license (http://creativecommons.org/licenses/by/3.0/). 